TEVATRON DAMPER SYSTEM

C. MOORE and ROGER RICE

DECEMBER 21， 1983 
Tevatron Damper System

C. Moore and R. Rice

A fast beam damper system is currently being built for the Tevatron. The system is similar to the super Damper system in the main ring with increased bandwidth and a pulsed high power mode for injection errors. The purpose of this writeup is to describe the basics of the system, and some of the reasoning behind its design. Calculations of expected performance are also included.

Since the damper has two jobs to perform, namely, beat down oscillations induced by injection errors and those due to instabilities at high intensity, we first need estimates of what injection errors will look like and what growth rates and frequencies to expect from instabilities.

Table 1 shows estimates for beam position errors due to injection devices assuming $\beta=100 \mathrm{~m}$. These values assumed pessimistic values for power supply regulation and neglected any long term drift. One can see that a few millimeters should be an upper limit for position errors due to injection line devices. In addition, structure of the order of a MHz may be present from the injection kicker.

For instabilities one can look at past experience with the Main Ring. 1 By simply comparing characteristics of the two machines which determine growth rates ${ }^{2}$ one can calculate an expected growth time for resistive wall instabilities in the Energy Doubler of about 10 times that for the Main Ring. Observed growth times in the Main Ring are tens of milliseconds, so for the Doubler we can expect growth times of the order of 100 msec.

similarly, if we compare parameters which influence the frequencies of these instabilities we find that we expect instabilities occurring at similar frequencies in both machines. Observed instabilities in the Main Ring start at around $35 \mathrm{kHz}$ (fractional tune $x$ revolution frequency). To damp instabilities on a bunch by bunch basis, of course, we need to operate at 53 MH z .

To summarize, what we are talking about so far is a system that can damp up to a few millimeter oscillations at injection, have a damping time under $100 \mathrm{msec}$ to suppress the growth of instabilities and handle frequencies from $20 \mathrm{kHz}-53 \mathrm{MHz}$. A more important damping time consideration is tune spread which determines how quickly the particles diffuse into a distribution larger than their initial distribution. Since the damper works on the centroid of the distribution it must work quickly compared to this diffusion time. For a nominal tune spread of .005, the beam diffuses in 200 turns or $4 \mathrm{msec}$. This means we really have 
to damp out injection errors in $1-2 \mathrm{msec}$ to prevent a large increase in emittance. Figure 1 shows a computer simulation of this diffusion process and the effect a damper can have on surpressing emittance growth. The plots are generated by a program written by King Yuen Bill Ng which will be discussed later.

The Damper system

The positions of each bunch will be measured by 1 meter long versions of the standard Doubler position detectors. The gain is similar to that of the standard short detector $(14.6 \mathrm{db} /$ in compared to $16.6 \mathrm{db} / \mathrm{in}$ ) but gives about 4 times the output for the same size beam pulse. The horizontal detector is located in the warm medium straight at Fl7 and the vertical detector is located in the $F \varnothing$ long straight at the downstream end as shown in Figure 2. Cabling from the detectors runs back to the R.F. building at $F \emptyset$. Two additional detectors at E48 will allow for damping of protons and $\bar{p}$ 's for colliding beam physics in the future.

The basic parameters of the deflectors are similar to the Main Ring Super Damper. The plates are $1.4 \mathrm{~m}$. long and have a gap of $6.4 \mathrm{~cm}$. The horizontal deflector will be at the Fll warm bypass and the vertical deflector will be at the upstream end of the $F \varnothing$ long straight. These locations give a nominal phase advance from the detectors of 19.25 for the vertical and 18.75 for the horizontal with a nominal machine tune of 19.4. The drivers for the deflectors will be housed in the Fø service building. They will supply up to $4 \mathrm{kV}$ during injection for up to $10 \mathrm{msec}$ and then supply a maximum of $1.4 \mathrm{kV}$ for the remainder of the cycle for instability suppression.

\section{Signal Processing}

The beam positions from the detectors will be digitized and delayed digitally to provide the deflection for the proper bunch. The signal is then converted back to an analog signal which is amplified and sent to the deflection plates. Detailed writeups on these systems will probably be forthcoming as they become operational.

\section{Damper Modeling}

The main tool used in determining the system parameters has been a simulation program written by King Yuen Bill $\mathrm{Ng}$. This program allows us to follow the time evolution of a group of particles (normally 2,000) with a given initial distribution as a Eunction of deflector plate length, deflector plate separation, deflector plate maximum voltage, system gain, tune spread, energy, mean tune, and phase separation between pickup and deflector. In general, we start with a uniform distribution in 
phase space with a given offset and ask for the amount of beam outside various $x$ adii centered in the beam pipe after a given number of turns. The size of the beam has been chosen to be .2 $\mathrm{cm}$ radius and the offsets range from .1 to $.5 \mathrm{~cm}$. Table 2 summarizes these parameters for the system.

Figure 3 shows the envelope of the centroid position as a function of time for three different cases. The upper curve is for no damping and with a tune spread of .005, as the beam spreads out the centroid position goes to zero in 200 turns or 4 msec. The other two pairs of curves show how quickly the centroid is damped to zero with $1.4 \mathrm{kV}$ and $4 \mathrm{kV}$ as a maximum plate voltage. The difference between the horizontal and vertical curves is due to the difference in betas at the detector and deflector locations.

Table 3 shows the results of modeling calculations in terms of emittance growth and damping time. One can see that the proposed system is expected to damp $2 \mathrm{~mm}$ injection errors with an emittance growth of only $50 \%$ in less than $1 \mathrm{msec}$.

\section{References}

1. R. Steining and E. J. N. Wilson, Nuclear Instruments and Methods 121,283 (1974).

2. B. zotter and F. Sacherer in Theoretical Aspects of the Behavior of Beams in Accelerators and Storage Rings, CERN 77-13 (1979). 


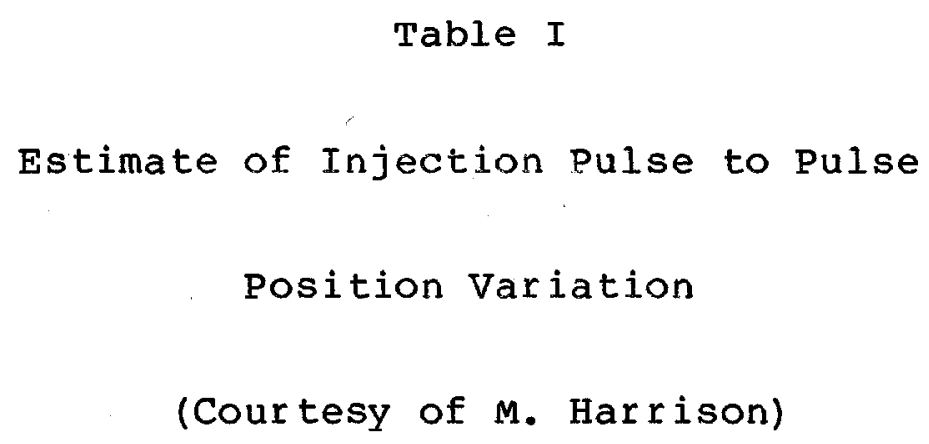

Vertical

Lambertson

M.R. position variation (meas.)

Horizontal

C26 - C32 - D38 Local Bump

D46 - El7 Local Bump

Injection Trim Magnets

M.R. Position Variations (meas.)

Horizontal Injection Kicker

(MHz structure)

Values neglect long term drift. $\pm .2 \mathrm{~mm}$

$\pm .1 \mathrm{~mm}$

$\pm .15 \mathrm{~mm}$

$\pm .15 \mathrm{~mm}$

$\pm .10 \mathrm{~mm}$

$\pm .65 \mathrm{~mm}$

$\pm 1.1 \mathrm{~mm}$ 
Table 2

\section{Damper Simulation Input Parameters}

Beam:
No. of particles: 2000
Distribution: Uniform
Init. Radius: $.2 \mathrm{~cm}$
Init. Emittance: $.04 \pi \mathrm{mm}-\mathrm{mr}$
Initial Displacement: $.1-.5 \mathrm{~cm}$
Energy $=150 \mathrm{GeV}$

Machine

$\beta$ at detectors $=60$ Meters Vertical; 100 Meters Horizontal

$\beta$ at deflectors $=100$ Meters Vertical; 80 Meters Horizontal

Tune $=19.4$

Oscillations between Monitor \& Deflector $=19.25$ Vertical;

18.75 Horizontal

Tune Spread: .005

Damper

Plate Length: $1.4 \mathrm{~m}$

Gap: $6.4 \mathrm{~cm}$

Gain: $100 \mathrm{kv} / \mathrm{cm}$ displacement

Max Voltage: $4 \mathrm{kv}$ during injection; $1.4 \mathrm{kv}$ for remainder of cycle 
Table 3

\section{Calculated Damper Performance}

$\Delta v= \pm .005 \Rightarrow$ chromaticity of 20

Emittance Growth

Initial offset (Inj. Error)

8 Increase in Emittance

$\begin{array}{rc}\text { proposed damper } & \text { no damper } \\ >400 & 1000 \\ 300 & 625 \\ 200 & 400 \\ 50 & 225 \\ 10 & \end{array}$

Damping Time

1/e Damping Time $=.3 \mathrm{msec}$

When saturated, damping rate $=3.0 \mathrm{~mm} / \mathrm{msec}$ at $4 . \mathrm{kv}$

$1.1 \mathrm{~mm} / \mathrm{msec}$ at $1.4 \mathrm{kv}$ 

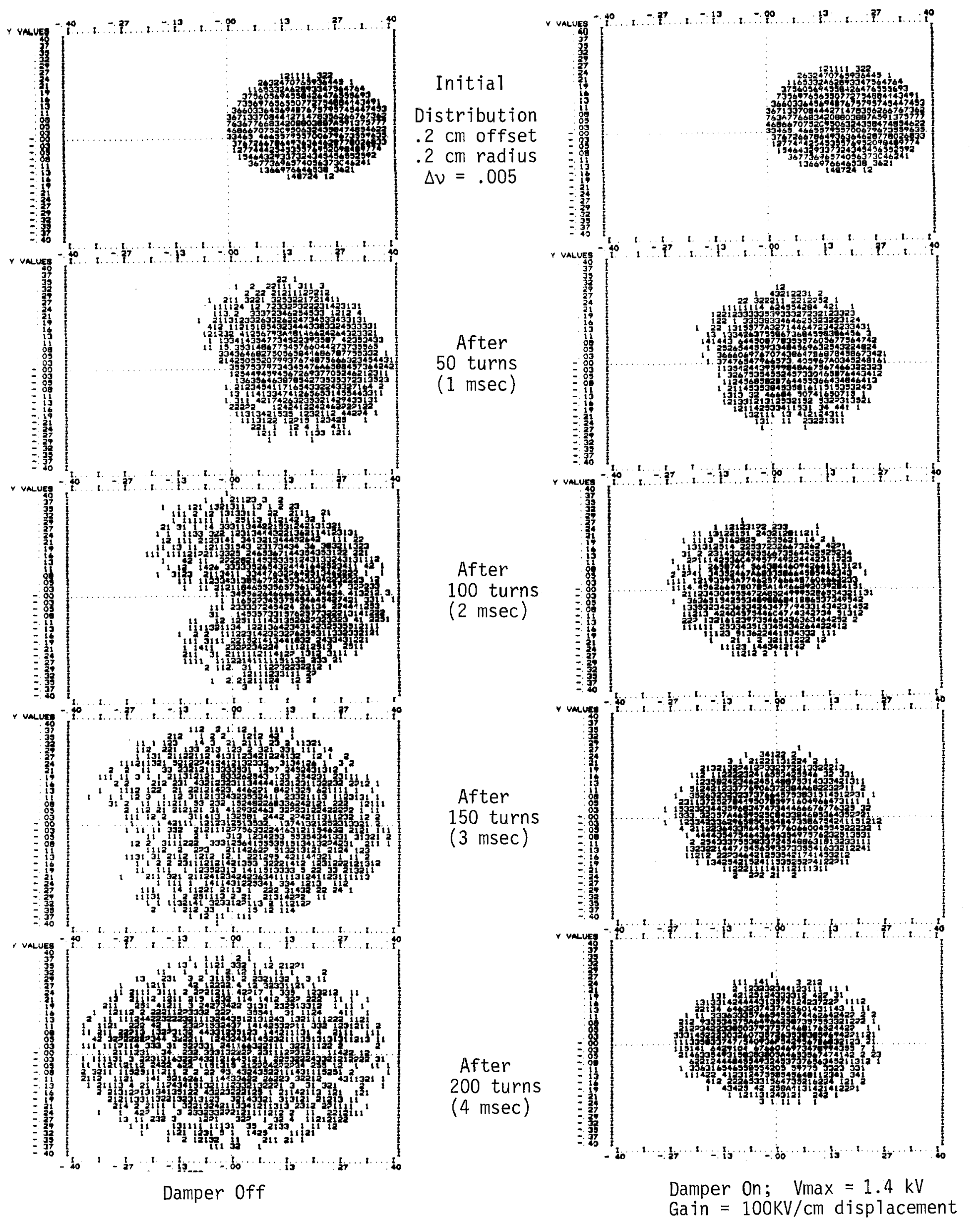

Figure 1: Beam distribution versus time for tune spread of .005 with damper on and off. 


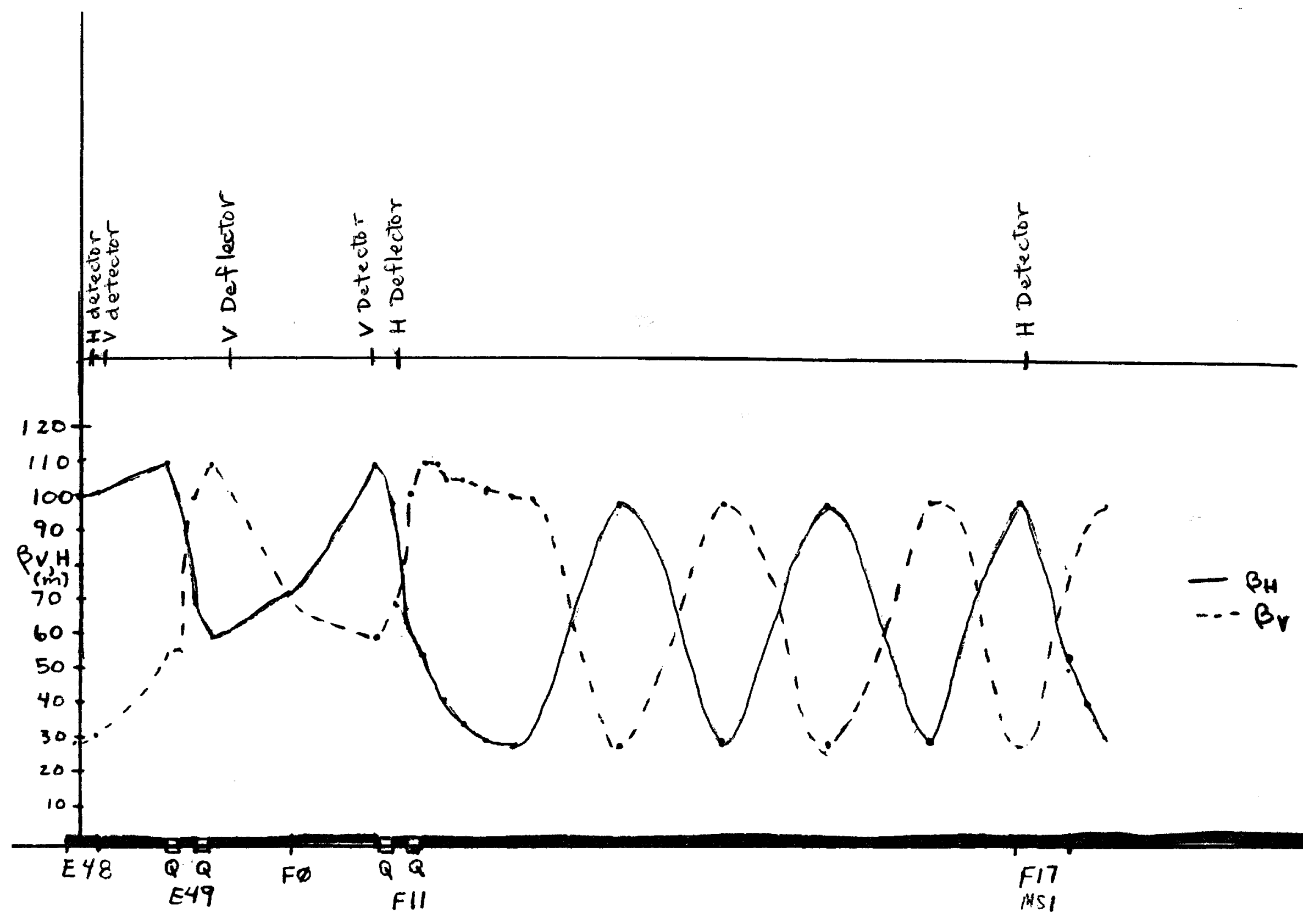

Figure 2: Tunne 1 location and $\beta^{\prime}$ 's of system components. 


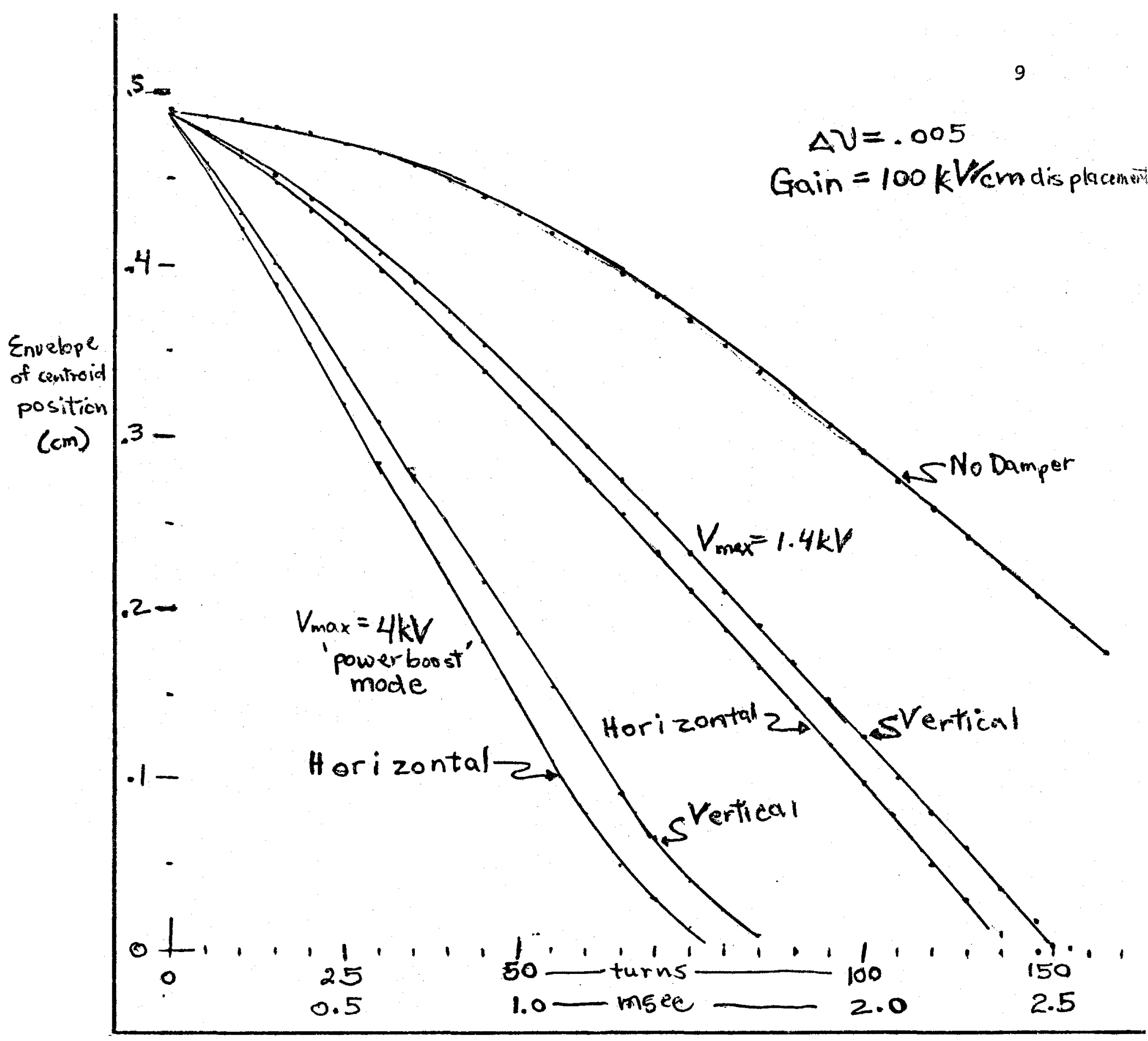

Figure 3: Computer simulation of beam centroid damping versus time for no damper, $V_{\max }=1.4 \mathrm{KV}$, and $V_{\max }=4.0 \mathrm{KV}$. 